\title{
Résumé de la déclaration concernant les voyageurs internationaux qui ont l'intention de rendre visite à des amis et à des parents
}

\author{
Brophy $\mathbf{J}^{1}$, au nom du Comité consultatif de la médecine tropicale et de la médecine des voyages \\ (CCMTMV)*
}

${ }^{1}$ Centre hospitalier pour enfants de l'est de l'Ontario, Ottawa (Ontario)

*Correspondance : CATMAT.Secretariat@phac-aspc.gc.ca

\section{Résumé}

Contexte : Les voyageurs rendant visite à des amis et à des parents constituent un groupe précis pour lequel on a déterminé qu'il court un risque accru de morbidité liée au voyage.

Objectif : Fournir des recommandations relatives à la réduction du risque des voyageurs internationaux rendant visite à des amis et à des parents.

Méthodologie : Les recommandations concernant les voyageurs rendant visite à des amis et à des parents des amis et parents ont été élaborées à partir de l'opinion d'experts du CCMTMV et de la littérature sur la médecine de voyage. Les risques précis liés au voyage, y compris l'épidémiologie des maladies infectieuses et leur fardeau dans cette population, ont été examinés et des recommandations ont été formulées dans le but d'essayer d'atténuer ces risques. On a fait référence à des déclarations précédentes du CCMTMV liées aux voyageurs rendant visite à des amis et à des parents, qui ont été réitérées.

Recommandations : Les taux des maladies liées aux voyages chez les voyageurs rendant visite à des amis et à des parents ont tendance à être plus élevés pour un grand nombre d'affections. La présente déclaration traite des recommandations et des facteurs de risque propres aux maladies. Le CCMTMV recommande que les vaccins des voyageurs rendant visite à des amis et à des parents soient à jour et que ces personnes reçoivent des conseils sur l'importance des différentes activités de réduction des risques, comme l'utilisation de la prophylaxie antipaludéenne, des pratiques sexuelles sécuritaires et la prévention des blessures. Le dépistage de la tuberculose avant et après le voyage est recommandé dans certaines situations.

Conclusion : L'évaluation de la santé avant le voyage est une occasion importante de s'attaquer aux problèmes des voyageurs rendant visite à des amis et à des parents liés aux croyances relatives à la santé, aux comportements en matière de santé, à l'état de santé actuel et à la possibilité de la présence d'affections préexistantes. Des discussions portant sur l'importance de l'observance des conseils en matière de santé et sur d'éventuels obstacles à l'observance peuvent être nécessaires.

\section{Préambule}

Le Comité consultatif de la médecine tropicale et de la médecine des voyages (CCMTMV) donne de façon continue à l'Agence de la santé publique du Canada des conseils opportuns de nature médicale, scientifique et sanitaire concernant les maladies tropicales infectieuses et les risques pour la santé associés aux voyages internationaux. L'Agence reconnaît que les recommandations et les conseils formulés dans cette déclaration reposent sur les pratiques médicales et les connaissances scientifiques les plus récentes et les diffuse dans le but d'informer les voyageurs ainsi que les professionnels de la santé qui sont appelés à leur prodiguer des soins. 
Les personnes qui administrent ou utilisent des médicaments, des vaccins ou d'autres produits devraient bien connaître la monographie des produits, ainsi que toute autre norme ou instruction approuvée concernant leur usage. Les recommandations relatives à l'usage des produits et les autres renseignements présentés ici peuvent différer de ceux qui figurent dans la monographie ou toute autre norme ou instruction approuvée pertinente établie par les fabricants autorisés. Les fabricants font approuver leurs produits et démontrent l'innocuité et l'efficacité de ceux-ci uniquement lorsque ces produits sont utilisés conformément à la monographie ou à toute autre norme ou instruction approuvée semblable.

\section{Introduction}

En 2012, rendre visite à des amis et à des parents était le deuxième motif le plus courant des voyages internationaux chez les voyageurs canadiens et représentait environ deux millions de visites avec nuitées dans des pays outre-mer (1). Les voyageurs rendant visite à des amis et à des parents constituent un groupe précis pour lequel on a déterminé qu'il court un risque accru de morbidité liée au voyage. Les Centers for Disease Control and Prevention des États-Unis définissent un voyageur rendant visite à des amis et à des parents comme « un immigrant, présentant une race et une ethnie différentes de celles de la majorité de la population de leur pays de résidence (un pays à revenu plus élevé), qui retourne dans son pays d'origine (pays à revenu plus faible) pour rendre visite à des amis ou à des parents. Les membres de la famille qui sont nés dans le pays de résidence, comme le conjoint ou les enfants, sont inclus dans la catégorie des voyageurs rendant visite à des amis et à des parents » (2).

Le présent document résume la déclaration du CCMTMV concernant les voyageurs internationaux rendant visite à des amis et à des parents qui présente une description complète des données probantes et des recommandations (3). La déclaration met l'accent sur la définition susmentionnée des voyageurs rendant visite à des amis et à des parents et elle décrit les risques accrus auxquels font face les voyageurs rendant visite à des amis et à des parents, tout en fournissant des recommandations en fonction de la documentation disponible. Étant donné les profils actuels de la mobilité de la population mondiale, cette définition des voyageurs rendant visite à des amis et à des parents peut être plutôt stricte. II est raisonnable d'étendre ces recommandations à tous les voyageurs qui se rendent dans des pays où il existe un gradient épidémiologique du risque par rapport au pays de résidence, dont l'intention est de rendre visite à des amis et à des parents, indépendamment de l'état d'immigrant ou de l'origine ethnique $(4,5)$.

\section{Méthodologie}

Une recherche documentaire a été réalisée pour les études publiées ainsi que pour la littérature grise. Les bases de données MEDLINE, EMBASE, Global Health, Scopus, Google Scholar et Access MEDLINE ont été utilisées ainsi que les rapports et les publications de Statistique Canada, de l'Agence de la santé publique du Canada (ASPC) et de Citoyenneté et Immigration Canada.

À partir de cet aperçu initial, des sujets de section ont été choisis et d'autres recherches documentaires ciblées ont été effectuées. Lorsqu'elles étaient disponibles, on a utilisé la littérature et les données probantes provenant du Canada en ce qui concerne l'épidémiologie et le fardeau de maladies précises dans la population des voyageurs rendant visite à des amis et à des parents. La déclaration ne contient pas un aperçu complet de tous les risques liés au voyage, puisque le contenu a été mis en ordre de priorité en fonction des risques qui sont particulièrement plus élevés pour les voyageurs rendant visite à des amis et à des parents. Par conséquent, il est important de bien connaître et de traiter tous les risques liés à la destination, en mettant un accent particulier sur les sujets abordés ci-dessous.

La déclaration consiste en un examen narratif de la littérature sur la médecine de voyage à propos des voyageurs rendant visite à des amis et à des parents et de l'opinion des experts du CCMTMV. Les recommandations ne comprennent pas une description de la fermeté de la recommandation ou de la qualité des données probantes, 
comme cela a été fait dans des déclarations précédentes du CCMTMV. On a fait référence à des déclarations précédentes du CCMTMV qui ont été réitérées lorsqu'elles étaient liées à des voyageurs rendant visite à des amis et à des parents et une liste complète des déclarations actuelles du CCMTMV se trouve sur le site Web de I'ASPC sur la santé des voyageurs (6).

\section{Résultats et recommandations}

\section{Facteurs de risque}

En raison de plusieurs facteurs, les taux des maladies liées aux voyages chez les voyageurs rendant visite à des amis et à des parents ont tendance à être plus élevés pour de nombreuses affections. Les voyageurs rendant visite à des amis et à des parents présentent une plus grande possibilité de plans de voyage à la dernière minute, ont souvent des séjours plus longs, peuvent être réticents à l'idée de manger différemment de leurs hôtes, peuvent demeurer dans des endroits sans moustiquaire de portes ou de fenêtres et sans moustiquaire de lit, se trouvent souvent en proximité immédiate avec la population locale et ont une probabilité accrue de consommation d'eau non traitée $(7,8)$.

Les voyageurs rendant visite à des amis et à des parents et les voyageurs nés à l'étranger sont moins susceptibles d'avoir recours à des consultations de santé avant le voyage $(9,10,11,12)$, et sont plus susceptibles d'avoir recours à des conseils à une date plus rapprochée du départ (13) et de refuser un vaccin recommandé (13). Ces différences ont été associées à la faible perception du risque de contracter personnellement la maladie chez les voyageurs rendant visite à des amis et à des parents (8), mais elles peuvent également refléter des obstacles langagiers, culturels ou financiers qui préviennent l'utilisation de ces services $(14,15)$. Les voyageurs rendant visite à des amis et à des parents croient souvent qu'ils sont immunisés contre les maladies (comme le paludisme) dans leur pays d'origine $(7,14,16)$. Ils peuvent demander des conseils de la part de fournisseurs de soins de santé provenant du même type de milieu ethnique qui peuvent ne pas recommander l'adoption de stratégies de prévention, comme la chimioprophylaxie, en raison de croyances semblables $(17,18)$.

\section{Paludisme}

Des études ont permis de noter que chez les voyageurs atteints de paludisme, $59 \%$ à $99 \%$ n'avaient pas eu recours à la chimioprophylaxie du paludisme ou l'avaient prise de façon inappropriée (médicament inapproprié ou observance inadéquate du traitement) (19). Dans une série de cas de diagnostics de paludisme au Canada, la vaste majorité des cas touchaient des voyageurs qui n'avaient pas demandé de conseils avant leur voyage ou qui n'avaient pas pris de prophylaxie antipaludéenne appropriée $(20,21)$.

Les voyageurs rendant visite à des amis et à des parents représentent une proportion importante des cas de paludisme importés dans les pays où la maladie n'est pas endémique (19). Des études ont révélé que les voyageurs nés à l'étranger rendant visite à des amis et à des parents présentent un risque jusqu'à 4,5 fois plus élevé de contracter le paludisme que les touristes (22). D'après les données de 2001 à 2013 du Réseau canadien sur le paludisme, $45 \%$ des cas graves de paludisme au Canada, pour lesquels de l'information était disponible, ont signalé que le but du voyage était de rendre visite à des amis et à des parents (23).

Étant donné les caractéristiques mentionnées ci-dessus qui peuvent augmenter le risque de paludisme (telles que la perception personnelle du risque de contracter la maladie et l'immunité), les voyageurs rendant visite à des amis et à des parents devraient recevoir des conseils sur l'importance de la prévention du paludisme lorsqu'ils voyagent dans des pays impaludés. Les recommandations doivent comprendre l'utilisation de mesures de protection individuelle pour prévenir les piqûres de moustiques, ainsi que l'utilisation potentielle de la chimioprophylaxie selon la destination. Les voyageurs rendant visite à des amis et à des parents devraient être avisés de demander des soins de santé s'ils font de la fièvre pendant le voyage ou après leur retour au Canada. 
Des recommandations précises sur la chimioprophylaxie du paludisme sont disponibles dans les

Recommandations canadiennes pour la prévention et le traitement du paludisme du CCMTMV (24).

\section{Maladies évitables par la vaccination}

En raison de la variation des calendriers de vaccination ou du manque d'accès à des vaccins dans différents pays, les immigrants canadiens peuvent être plus à risque de contracter des maladies évitables par la vaccination.

\section{Immunisation systématique}

Des études ont révélé des taux importants de non-immunisation contre la rougeole, les oreillons, la rubéole et la varicelle chez les immigrants canadiens en provenance de pays en développement $(25,26,27)$. II faudrait évaluer l'état immunitaire et l'immunité des voyageurs rendant visite à des amis et à des parents contre les maladies évitables par la vaccination, et des vaccinations systématiques devraient être fournies au besoin. Pour les jeunes voyageurs rendant visite à des amis et à des parents, il peut y avoir une possibilité d'accélérer le calendrier de vaccination systématique en vue d'offrir une protection maximale pendant le voyage.

L'annexe 2 de la version intégrale de la Déclaration concernant les voyageurs rendant visite à des amis et à des parents (3) et la Déclaration relative aux jeunes voyageurs du CCMTMV (28) contiennent des renseignements sur les calendriers de vaccination accélérés chez les enfants.

\section{Typhoïde}

La majorité des cas de fièvre typhoïde en Amérique du Nord sont associés aux voyages, en particulier à destination d'Asie du Sud (Afghanistan, Pakistan, Inde, Népal, Bangladesh, Maldives, Sri Lanka et Bhoutan) (15). Des études ont révélé que les voyageurs rendant visite à des amis et à des parents constituent un facteur de risque important de fièvre typhoïde liée aux voyages, puisqu'ils représentent $66 \%$ des cas aux États-Unis (29) et plus de $90 \%$ des cas au Québec (30). Une étude menée par le réseau mondial GeoSentinel a révélé que les voyageurs rendant visite à des amis et à des parents présentent un risque 7 fois plus élevé de recevoir un diagnostic de fièvre typhoïde que les touristes (22).

La vaccination contre la typhoïde adaptée à l'âge est recommandée pour les voyageurs rendant visite à des amis et à des parents en Asie du Sud (31). Le vaccin contre la typhoïde n'est pas systématiquement recommandé pour les voyageurs qui se rendent dans des destinations à l'extérieur de l'Asie du Sud; cependant, il peut être envisagé pour les voyageurs rendant visite à des amis et à des parents dans des situations particulières à risque élevé (31). Les précautions en matière de consommation d'eau et d'aliments devraient faire l'objet d'une discussion, et l'accent devrait être mis sur l'importance du lavage fréquent des mains.

La Déclaration concernant les voyageurs internationaux et la typhoïde (31) du CCMTMV offre de plus amples renseignements sur la prévention de la fièvre typhoïde et l'utilisation du vaccin contre la typhoïde.

\section{Hépatite A et hépatite $B$}

Les voyageurs rendant visite à des amis et à des parents, surtout les enfants, sont l'une des principales causes des cas d'hépatite A déclarés en Europe et en Amérique du Nord. Des études européennes et québécoises ont révélé que les voyageurs rendant visite à des amis et à des parents représentent $28 \%$ à $78 \%$ des cas d'hépatite A liés aux voyages $(30,32,33,34,35)$.

Des recherches ont révélé que plus de la moitié des immigrants et des réfugiés ne sont pas immunisés contre I'hépatite B (36). Plusieurs caractéristiques comportementales des voyageurs rendant visite à des amis et à des parents (périodes plus longues dans le pays, contacts étroits avec la population locale, plus grand risque de blessure ou contact avec le système médical) et les taux élevés de non-immunisation devraient être considérés comme des facteurs de risque précis pour l'acquisition de l'hépatite B. 
Les personnes qui voyagent dans des pays où les conditions de salubrité et d'hygiène sont mauvaises devraient être avisées d'employer des précautions en matière de salubrité des aliments et de l'eau et de se laver les mains fréquemment (37). Les voyageurs rendant visite à des amis et à des parents dans des pays où l'hépatite $B$ est endémique ou qui pourraient adopter des comportements augmentant leur risque de contact avec du sang et des liquides organiques doivent recevoir des conseils concernant les pratiques sécuritaires (utilisation du condom, utilisation d'équipement médical stérile) (38). Les voyageurs qui ne sont pas immunisés contre le virus de I'hépatite $A$ et de l'hépatite $B$ devraient être vaccinés avant le voyage (37). Une immunisation adaptée à l'âge est recommandée pour les enfants.

De plus amples recommandations sur la prévention de l'hépatite $A$ et de l'hépatite $B$ chez les voyageurs se trouvent dans le Résumé des recommandations pour la prévention de la contraction d'hépatites virales en voyage du CCMTMV (37).

\section{Tuberculose}

Les voyageurs qui se rendent dans des pays où l'incidence de la tuberculose (TB) est plus élevée sont à risque de contracter l'infection durant le voyage. Les personnes nées à l'étranger représentaient $64 \%$ de tous les cas signalés au Canada en 2012, et les taux d'incidence les plus élevés ont été observés chez les personnes venant de l'Afrique, de l'Asie du Sud-Est, du Pacifique occidental et de la Méditerranée orientale (39). Ces cas comprennent des cas de tuberculose contractée dans le pays d'origine avant l'immigration, de même que durant des voyages visant à rendre visite à des amis et à des parents. Des études ont révélé que des proportions importantes de cas de tuberculose chez les populations d'immigrants peuvent être attribuées à des voyages visant à rendre visite à des amis et à des parents $(40,41)$. En outre, les voyages à l'étranger, particulièrement les voyages visant à rendre visite à des amis et à des parents, ont été établis comme un facteur de risque de l'infection tuberculeuse latente (ITL) $(42,43)$.

Les voyageurs rendant visite à des amis et à des parents dans des pays où l'incidence de la tuberculose est élevée devraient éviter la consommation de produits laitiers non pasteurisés afin de réduire le risque de contracter $M$. bovis (44). Les voyageurs rendant visite à des amis et à des parents devraient être mis en garde d'éviter les personnes atteintes de tuberculose pulmonaire connue ou d'une toux chronique inexpliquée jusqu'à ce que la personne soit jugée non contagieuse. Le vaccin bacille de Calmette-Guérin (BCG) peut être envisagé dans certaines circonstances pour les voyageurs qui se rendent à long terme dans des pays où la prévalence de la tuberculose est élevée (45).

Des tests cutanés de dépistage de la tuberculose avant et après le voyage peuvent être indiqués selon les risques que présente la destination, la durée du voyage et les facteurs de santé personnelle. La Déclaration concernant les voyageurs rendant visite à des amis et à des parents, version intégrale, contient un modèle décisionnel pour orienter le test cutané de dépistage de la tuberculose chez les voyageurs (3).

Le lecteur est prié de consulter l'Évaluation du risque de tuberculose et prévention de cette maladie chez les voyageurs (44) pour des lignes directrices détaillées sur les conseils offerts avant un voyage pour éviter un risque d'infection tuberculeuse et dépister la tuberculose après un voyage ainsi que les Normes canadiennes pour la lutte antituberculeuse (45) pour obtenir des renseignements à jour sur les conditions à risque de tuberculose et le niveau de stratification du risque des pays.

\section{Infections parasitaires}

Certaines infections parasitaires, telles que la schistosomiase, la strongyloidïose, l'échinococcose et la cysticercose, peuvent être chroniques et peuvent entraîner une morbidité importante, voire la mort, tandis que d'autres sont résolutives et ont une faible incidence sur la santé. Une étude GeoSentinel canadienne a révélé que les voyageurs rendant visite à des amis et à des parents sont plus susceptibles de présenter ces infections parasitaires et d'autres (46). 
Les voyageurs rendant visite à des amis et à des parents devraient être avisés d'éviter les activités en eau douce (telles que la baignade) dans les régions où la schistosomiase est endémique (Afrique, Asie du Sud-Est et certaines régions d'Amérique du Sud). On recommande d'éviter les contacts entre la peau et le sol (comme la marche pieds nus) dans les pays tropicaux afin de prévenir la strongyloidïose. On recommande des précautions avant de consommer de l'eau et des aliments afin de prévenir la propagation d'autres infections parasitaires par voie oro-fécale. L'évaluation avant le voyage peut offrir une occasion de déterminer les risques et de recommander le dépistage de ces infections parasitaires chroniques traitables chez les immigrants.

\section{Infections transmissibles sexuellement et virus de l'immunodéficience humaine}

Des relations sexuelles non protégées et de nouveaux partenaires sexuels sont courants chez les voyageurs en général (47), y compris les voyageurs rendant visite à des amis et à des parents $(48,49)$. Un récent examen mondial GeoSentinel a révélé que les voyages ayant pour but de rendre visite à des amis et à des parents sont associés à un risque accru d'infections transmissibles sexuellement (ITS) par rapport aux autres voyages (50). Cette constatation s'ajoute à la documentation existante qui indique que les ITS sont plus probables chez les voyageurs rendant visite à des amis et à des parents que chez les autres voyageurs $(22,51)$.

Des conseils en matière de santé sexuelle devraient systématiquement faire partie de la consultation avant le voyage et le risque d'exposition aux ITS et au virus de l'immunodéficience humaine (VIH) devrait faire l'objet d'une discussion. II faut insister sur l'importance d'adopter des pratiques sexuelles plus sécuritaires et les voyageurs devraient être encouragés à apporter des condoms du Canada pour en assurer leur qualité (52). Le vaccin contre le VPH peut être envisagé pour les voyageurs adolescents et adultes qui n'ont pas déjà été vaccinés. Le vaccin contre l'hépatite $B$ est recommandé comme il est indiqué ci-dessus.

Le lecteur est prié de consulter la Déclaration sur les voyageurs et les infections transmissibles sexuellement (52) pour obtenir d'autres recommandations liées à la réduction du risque d'ITS.

\section{Blessure}

Les blessures constituent une cause importante de morbidité et de mortalité chez les voyageurs et représentent de $18 \%$ à $25 \%$ des cas de mortalité des voyageurs à l'étranger $(53,54,55)$. On présume que de nombreuses caractéristiques des voyageurs rendant visite à des amis et à des parents (plus longue durée de séjour, plus grande probabilité d'utilisation de modes de transport locaux) augmentent le risque de blessures, même s'il n'existe aucune documentation précise sur les blessures liées aux voyages chez les voyageurs rendant visite à des amis et à des parents.

Des précautions de sécurité routière comme le port de la ceinture de sécurité, l'utilisation de sièges d'auto pour nourrissons et pour enfants et le port d'un casque devraient être recommandées (56). Les voyageurs rendant visite à des amis et à des parents devraient éviter l'utilisation de motocyclettes ou de bicyclettes et devraient être encouragés à porter un casque s'ils ne peuvent éviter ces moyens de transport (56). Pour obtenir de plus amples renseignements sur le risque de blessure et les recommandations en matière de prévention, le lecteur est prié de consulter la Déclaration sur les risques de blessure chez les voyageurs du CCMTMV (56).

\section{Populations spéciales}

\section{Jeunes voyageurs rendant visite à des amis et à des parents}

Comparativement aux jeunes touristes, les jeunes voyageurs rendant visite à des amis et à des parents sont plus susceptibles d'être plus jeunes, de voyager pendant de plus longues périodes, de voyager plus souvent dans des régions rurales, de se présenter plus près de la date de départ pour obtenir des conseils avant le voyage et de se rendre dans des destinations à risque plus élevé de maladies tropicales $(57,58)$.

Les enfants rendant visite à des amis et à des parents présentent un risque accru de contracter des maladies liées aux voyages et sont particulièrement à risque de maladie fébrile (surtout causée par le paludisme) (59), de 
tuberculose, de typhoïde et de méningite à méningocoques (60). Une récente étude de surveillance des jeunes voyageurs rendant visite à des amis et à des parents du Canada a révélé que la fièvre entérique, le paludisme, les maladies diarrhéiques et l'hépatite A représentaient $75 \%$ des maladies liées aux voyages dans ce groupe (61). Seulement $26 \%$ de ces voyageurs avaient reçu des conseils avant leur voyage.

Ces résultats mettent l'accent sur l'importance de l'évaluation effectuée avant un voyage et de l'observance des interventions recommandées pour les enfants. Les possibilités pour accélérer le calendrier de vaccination systématique devraient être évaluées afin de fournir une protection maximale pendant un voyage (se reporter à l'annexe 2 de la version intégrale de la Déclaration concernant les voyageurs rendant visite à des amis et à des parents (3) pour les calendriers de vaccination accélérés). Les parents devraient être informés que les taux de maladie nécessitant une hospitalisation sont plus élevés chez les jeunes voyageurs rendant visite à des amis et à des parents et qu'une maladie apparaissant pendant et après un voyage exige une évaluation urgente. De plus, les parents de jeunes voyageurs nés au Canada rendant visite à des amis et à des parents devraient recevoir des conseils à propos du fait que leur enfant ne possède pas d'immunité innée contre les maladies liées aux voyages en raison de son identité génétique seulement. Pour obtenir de plus amples renseignements sur les jeunes voyageurs ainsi que des recommandations, le lecteur est prié de consulter la Déclaration relative aux jeunes voyageurs du CCMTMV (28).

\section{Sujets immunodéprimés et voyageurs âgés rendant visite à des amis et à des parents}

Peu de recherches sont axées sur les sujets immunodéprimés ou les voyageurs âgés rendant visite à des amis et à des parents. Certaines caractéristiques particulières de ces populations (maladies comorbides, réponses immunitaires plus faibles, contre-indications liées aux vaccins ou fragilité) qui augmentent les risques pour la santé en voyage peuvent entraîner des risques synergiques pour les voyageurs rendant visite à des amis et à des parents.

Les risques de maladies infectieuses doivent être examinés auprès du voyageur selon son degré d'immunodépression ainsi que les risques propres à la destination et le caractère approprié du voyage.

Pour des renseignements sur la santé des voyageurs immunodéprimés ainsi que des recommandations détaillées sur des affections précises, le lecteur est prié de consulter la déclaration sur Le voyageur immunodéprimé du CCMTMV (62). Pour obtenir des renseignements sur les voyageurs âgés ainsi que des recommandations à leur intention, le lecteur est prié de consulter la Déclaration sur les voyageurs âgés du CCMTMV (63).

\section{Ciblage des voyageurs rendant visite à des amis et à des parents pour des conseils avant leur} voyage

Lors des visites régulières, les fournisseurs de soins de santé doivent discuter avec leurs patients d'éventuels voyages dans le but de rendre visite à des amis et à des parents. La consultation d'un spécialiste de la santé des voyageurs devrait être recommandée pour tous les voyageurs rendant visite à des amis et à des parents, et plus particulièrement pour les personnes présentant des facteurs de risque de maladie grave. Étant donné que certains patients peuvent ne pas vouloir ou pouvoir payer le coût de la consultation en médecine de voyage, les fournisseurs de soins primaires doivent posséder des connaissances et des ressources cliniques en matière de santé des voyageurs de façon à ce qu'ils soient en mesure de fournir des recommandations essentielles appropriées.

\section{Conclusion}

La consultation avant le voyage pour les voyageurs rendant visite à des amis et à des parents fournit une occasion de promotion de la santé, de détermination des affections préexistantes et de réduction des risques. Les voyageurs rendant visite à des amis et à des parents devraient être mis au courant de leur risque accru de 
maladies liées aux voyages et de la façon de prévenir ces dernières. Les taux élevés de non-immunité à des maladies évitables par la vaccination et l'augmentation de la prévalence des maladies chroniques chez les voyageurs rendant visite à des amis et à des parents devraient également être abordés. En outre, les fournisseurs de soins de santé devraient insister sur l'importance de l'observance des recommandations et aborder les défis potentiels afin d'atteindre cet objectif.

Le Tableau 1 résume les recommandations propres à chaque maladie à l'intention des voyageurs rendant visite à des amis et à des parents ainsi que d'autres ressources. D'autres recherches sont nécessaires pour déterminer les facilitateurs et les obstacles à l'accès aux conseils offerts avant le voyage ainsi qu'à leur observance pour les voyageurs rendant visite à des amis et à des parents. La participation des communautés ethniques et du personnel de soins de santé qui leur fournissent des soins est nécessaire pour évaluer les connaissances, les attitudes et les comportements en matière de santé des voyageurs et pour déterminer les meilleures façons de fournir de l'information aux voyageurs rendant visite à des amis et à des parents.

Tableau 1 : Recommandations propres à chaque maladie pour les voyageurs rendant visite à des amis et à des parents et ressources supplémentaires

\begin{tabular}{|c|c|c|}
\hline Maladie & $\begin{array}{l}\text { Stratégies visant à réduire les risques } \\
\text { associés aux voyages des voyageurs } \\
\text { rendant visite à des amis et à des parents }\end{array}$ & $\begin{array}{l}\text { Ressources pour de plus } \\
\text { amples renseignements }\end{array}$ \\
\hline Paludisme & 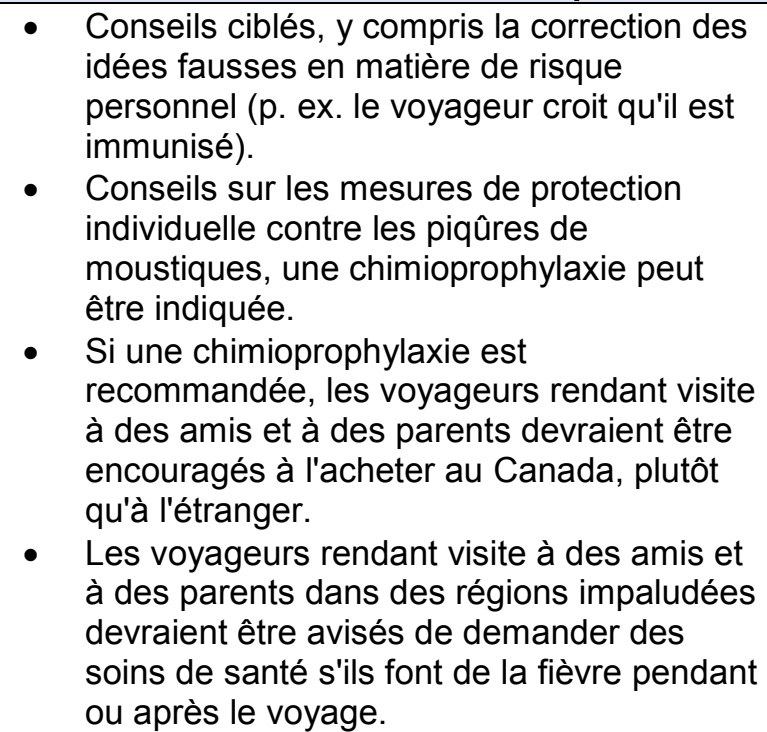 & $\begin{array}{l}\text { Recommandations } \\
\text { canadiennes pour la } \\
\text { prévention et le traitement } \\
\text { du paludisme du CCMTMV } \\
\text { (24) } \\
\text { Déclaration relative aux } \\
\text { mesures de protection } \\
\text { individuelle pour prévenir } \\
\text { les piqûres ou morsures } \\
\text { d'arthropodes du CCMTMV } \\
\text { (64) } \\
\text { Enfants : Déclaration } \\
\text { relative aux jeunes } \\
\text { voyageurs du CCMTMV } \\
\text { (28) }\end{array}$ \\
\hline $\begin{array}{l}\text { Maladies évitables } \\
\text { par la vaccination } \\
\text { systématique }\end{array}$ & $\begin{array}{l}\text { - Évaluer l'état immunitaire des personnes } \\
\text { nées à l'étranger et mettre à jour leurs } \\
\text { vaccinations systématiques, au besoin. } \\
\text { - Enfants : Une possible accélération de la } \\
\text { primovaccination devrait être étudiée afin } \\
\text { de fournir une protection maximale pendant } \\
\text { le voyage. }\end{array}$ & $\begin{array}{l}\text { - Annexe } 2 \text { de la version } \\
\text { intégrale de la Déclaration } \\
\text { concernant les voyageurs } \\
\text { rendant visite à des amis et } \\
\text { à des parents, tableau des } \\
\text { calendriers de vaccination } \\
\text { accélérés chez les enfants } \\
\text { (3) } \\
\text { - Déclaration relative aux } \\
\text { jeunes voyageurs du } \\
\text { CCMTMV (28) } \\
\text { Partie } 3 \text { du Guide canadien } \\
\text { d'immunisation, }\end{array}$ \\
\hline
\end{tabular}




\begin{tabular}{|c|c|c|}
\hline & & $\begin{array}{l}\text { Immunisation des } \\
\text { voyageurs }(65)\end{array}$ \\
\hline Typhoïde & $\begin{array}{l}\text { - Les personnes qui voyagent dans des pays } \\
\text { où les conditions de salubrité et d'hygiène } \\
\text { sont mauvaises devraient être avisées } \\
\text { d'employer des précautions en matière de } \\
\text { salubrité des aliments et de l'eau et de se } \\
\text { laver les mains fréquemment. } \\
\text { - Le vaccin contre la typhoïde est } \\
\text { recommandé pour les adultes et les enfants } \\
\text { (lorsqu'il est approprié à l'âge de l'enfant) } \\
\text { qui voyagent en Asie du Sud (31). } \\
\text { Le vaccin contre la typhoïde n'est pas } \\
\text { systématiquement recommandé pour les } \\
\text { voyageurs qui se rendent dans des } \\
\text { destinations autres que l'Asie du Sud'; } \\
\text { cependant, il peut être envisagé pour les } \\
\text { voyageurs rendant visite à des amis et à } \\
\text { des parents dans des situations qui posent } \\
\text { un risque important (p. ex. les enfants, de } \\
\text { longues périodes de séjour, l'impossibilité } \\
\text { d'éviter les expositions } \\
\text { alimentaires/hydriques à risque élevé) (31). }\end{array}$ & $\begin{array}{l}\text { Déclaration du CCMTMV } \\
\text { concernant les voyageurs } \\
\text { internationaux et la fièvre } \\
\text { typhoïde (31) }\end{array}$ \\
\hline Hépatite $A$ & $\begin{array}{l}\text { - Les voyageurs devraient être avisés } \\
\text { d'employer des précautions en matière de } \\
\text { salubrité des aliments et de l'eau et de se } \\
\text { laver les mains fréquemment. } \\
\text { - Les voyageurs non immunisés rendant } \\
\text { visite à des amis et à des parents dans des } \\
\text { pays en développement devraient être } \\
\text { vaccinés. } \\
\text { - Enfant : Le vaccin contre l'hépatite A } \\
\text { approprié à l'âge est recommandé pour les } \\
\text { enfants. }\end{array}$ & 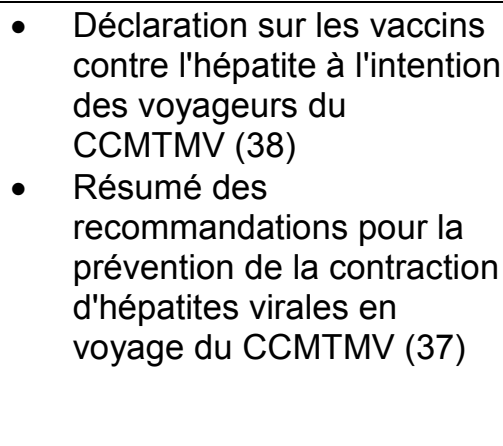 \\
\hline Hépatite $B$ & $\begin{array}{l}\text { Tous les voyageurs rendant visite à des } \\
\text { amis et à des parents dans des pays où } \\
\text { l'hépatite B est endémique (c.-à-d. } \\
\text { présentant une prévalence de l'antigène de } \\
\text { surface de l'hépatite B } \geq 2 \% \text { ) ou qui } \\
\text { pourraient adopter des comportements } \\
\text { augmentant leur risque de contact avec du } \\
\text { sang ou des liquides organiques doivent } \\
\text { recevoir des conseils en matière de } \\
\text { pratiques sécuritaires (utilisation du } \\
\text { condom, utilisation de matériel médical } \\
\text { stérile). } \\
\text { Les voyageurs non immunisés rendant } \\
\text { visite à des amis et à des parents devraient } \\
\text { être vaccinés. } \\
\text { Enfant : Le vaccin contre l'hépatite B } \\
\text { approprié à l'âge est recommandé pour les }\end{array}$ & $\begin{array}{l}\text { - Déclaration sur les vaccins } \\
\text { contre l'hépatite à l'intention } \\
\text { des voyageurs du } \\
\text { CCMTMV (38) } \\
\text { Résumé des } \\
\text { recommandations pour la } \\
\text { prévention de la contraction } \\
\text { d'hépatites virales en } \\
\text { voyage du CCMTMV (37) } \\
\text { Carte des pays } \\
\text { endémiques de } \\
\text { l'Organisation mondiale de } \\
\text { la Santé (66) }\end{array}$ \\
\hline
\end{tabular}




\begin{tabular}{|c|c|c|}
\hline & enfants. & \\
\hline Tuberculose & $\begin{array}{l}\text { - Les voyageurs rendant visite à des amis et } \\
\text { à des parents devraient éviter tout contact } \\
\text { avec des personnes atteintes d'une } \\
\text { tuberculose pulmonaire connue (pendant } \\
\text { qu'elles sont contagieuses) ou d'une toux } \\
\text { chronique inexpliquée. } \\
\text { Des tests cutanés de dépistage de la } \\
\text { tuberculose avant et après le voyage } \\
\text { peuvent être indiqués selon les risques que } \\
\text { présente la destination, la durée du voyage } \\
\text { et les facteurs de santé personnelle. La } \\
\text { version intégrale de la Déclaration } \\
\text { concernant les voyageurs rendant visite à } \\
\text { des amis et à des parents contient un } \\
\text { modèle décisionnel pour orienter le test } \\
\text { cutané de dépistage de la tuberculose chez } \\
\text { les voyageurs (3). } \\
\text { Dans certaines circonstances } \\
\text { exceptionnelles, le vaccin bacille de } \\
\text { Calmette-Guérin peut être envisagé pour } \\
\text { les voyageurs qui se rendent à long terme } \\
\text { dans des pays où la prévalence de la } \\
\text { tuberculose est élevée. } \\
\text { Les voyageurs rendant visite à des amis et } \\
\text { à des parents dans des pays où l'incidence } \\
\text { de la tuberculose est élevée devraient } \\
\text { éviter la consommation de produits laitiers } \\
\text { non pasteurisés afin d'éviter le risque de } \\
\text { contracter } M \text {. bovis. }\end{array}$ & $\begin{array}{l}\text { - Annexes } 3,4 \text { et } 5 \text {, les } \\
\text { critères de test cutané de } \\
\text { dépistage de la tuberculose } \\
\text { après le voyage, les } \\
\text { facteurs de risque pour le } \\
\text { développement de la } \\
\text { tuberculose active et le } \\
\text { modèle décisionnel pour } \\
\text { orienter le test cutané de } \\
\text { dépistage de la tuberculose } \\
\text { (3) } \\
\text { Évaluation du risque de } \\
\text { tuberculose et prévention } \\
\text { de cette maladie chez les } \\
\text { voyageurs du CCMTMV } \\
\text { (44) } \\
\text { Normes canadiennes pour } \\
\text { la lutte antituberculeuse } \\
\text { (chapitres } 6 \text { et 13) (45) } \\
\text { Enfants : Déclaration } \\
\text { relative aux jeunes } \\
\text { voyageurs du CCMTMV } \\
\text { (28) }\end{array}$ \\
\hline $\begin{array}{l}\text { Infections } \\
\text { parasitaires }\end{array}$ & $\begin{array}{l}\text { - Les voyageurs devraient être avisés } \\
\text { d'éviter les activités en eau douce, telles } \\
\text { que la baignade, en Afrique, en Asie du } \\
\text { Sud-Est et dans certaines régions de } \\
\text { l'Amérique du Sud pour prévenir la } \\
\text { schistosomiase. } \\
\text { Éviter de marcher pieds nus ou d'avoir } \\
\text { d'autres contacts entre la peau et le sol } \\
\text { dans les pays tropicaux pour prévenir la } \\
\text { strongyloidiose. } \\
\text { Suivre les précautions en matière de } \\
\text { salubrité des aliments et de l'eau pour la } \\
\text { prévention des diverses infections } \\
\text { parasitaires intestinales. }\end{array}$ & \\
\hline $\begin{array}{l}\text { Infections } \\
\text { transmissibles } \\
\text { sexuellement (ITS) } \\
\text { et virus de } \\
\text { l'immunodéficience } \\
\text { humaine (VIH) }\end{array}$ & $\begin{array}{l}\text { Discuter de la possibilité d'activités } \\
\text { sexuelles au cours du voyage, ainsi que } \\
\text { des taux d'ITS et de VIH dans la population } \\
\text { générale et la population des travailleurs du } \\
\text { sexe à destination. } \\
\text { - Insister sur l'importance d'adopter des } \\
\text { pratiques sexuelles plus sécuritaires et sur }\end{array}$ & $\begin{array}{l}\text { Déclaration sur les } \\
\text { voyageurs et les infections } \\
\text { transmissibles } \\
\text { sexuellement du CCMTMV } \\
\text { (52) }\end{array}$ \\
\hline
\end{tabular}




\begin{tabular}{|c|c|c|}
\hline & $\begin{array}{l}\text { la préparation en prévision d'un voyage en } \\
\text { apportant des condoms du Canada (pour } \\
\text { en assurer leur qualité). } \\
\text { - Le vaccin contre l'hépatite B devrait être } \\
\text { recommandé comme il est indiqué ci- } \\
\text { dessus. } \\
\text { - Le vaccin contre le VPH peut être envisagé } \\
\text { pour les voyageurs adolescents et adultes } \\
\text { qui n'ont pas déjà été vaccinés. }\end{array}$ & \\
\hline Blessure & $\begin{array}{l}\text { - Les voyageurs rendant visite à des amis et } \\
\text { à des parents devraient éviter l'utilisation de } \\
\text { motocyclettes ou de bicyclettes et porter un } \\
\text { casque si ces activités ne peuvent être } \\
\text { évitées. } \\
\text { Encourager les mesures de précautions en } \\
\text { matière de sécurité routière comme le port } \\
\text { de la ceinture de sécurité et l'utilisation de } \\
\text { sièges d'auto pour les nourrissons et les } \\
\text { enfants. }\end{array}$ & $\begin{array}{l}\text { Déclaration sur les risques } \\
\text { de blessure chez les } \\
\text { voyageurs du CCMTMV } \\
\text { (56) }\end{array}$ \\
\hline Jeunes voyageurs & $\begin{array}{l}\text { - Conseiller les parents à propos du fait que } \\
\text { les taux de maladie nécessitant une } \\
\text { hospitalisation sont plus élevés chez les } \\
\text { jeunes voyageurs rendant visite à des amis } \\
\text { et à des parents et que l'apparition d'une } \\
\text { maladie pendant et après un voyage exige } \\
\text { une évaluation urgente. } \\
\text { - Aborder les idées fausses concernant } \\
\text { l'immunité (p. ex., l'enfant n'est pas protégé } \\
\text { parce que ses parents sont nés dans le } \\
\text { pays de destination). } \\
\text { - Il peut être possible d'accélérer la } \\
\text { vaccination. } \\
\text { Le test cutané de dépistage de la } \\
\text { tuberculose après le voyage et le vaccin } \\
\text { bacille de Calmette-Guérin peuvent être } \\
\text { indiqués dans certaines situations; } \\
\text { consulter les ressources supplémentaires } \\
\text { pour les recommandations. }\end{array}$ & $\begin{array}{l}\text { - } \text { Recommandations propres } \\
\text { aux enfants dans les } \\
\text { sections précédentes, y } \\
\text { compris dans les } \\
\text { Recommandations } \\
\text { canadiennes pour la } \\
\text { prévention et le traitement } \\
\text { du paludisme du CCMTMV } \\
\text { (24) } \\
\text { Annexe } 2 \text { pour un } \\
\text { calendrier de vaccination } \\
\text { accéléré (3) } \\
\text { Déclaration relative aux } \\
\text { jeunes voyageurs du } \\
\text { CCMTMV (28) } \\
\text { Évaluation du risque de } \\
\text { tuberculose et prévention } \\
\text { de cette maladie chez les } \\
\text { voyageurs du CCMTMV } \\
\text { (44) } \\
\text { Normes canadiennes pour } \\
\text { la lutte antituberculeuse } \\
\text { (chapitres } 6 \text { et 13) (45) }\end{array}$ \\
\hline Voyageurs âgés & $\begin{array}{l}\text { - Il faudrait effectuer un examen des } \\
\text { comorbidités et formuler des } \\
\text { recommandations en fonction de l'âge. } \\
\text { - Un test cutané de dépistage de la } \\
\text { tuberculose avant ou après le voyage peut } \\
\text { être indiqué. }\end{array}$ & 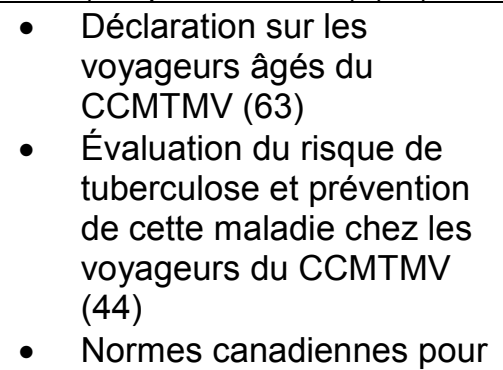 \\
\hline
\end{tabular}




\begin{tabular}{|c|c|c|}
\hline & & $\begin{array}{l}\text { la lutte antituberculeuse } \\
\text { (chapitres } 6 \text { et 13) (45) }\end{array}$ \\
\hline $\begin{array}{l}\text { Voyageurs } \\
\text { immunodéprimés }\end{array}$ & $\begin{array}{l}\text { Examiner les risques d'infection pour les } \\
\text { voyageurs selon l'immunodépression } \\
\text { personnelle et les risques à destination et } \\
\text { discuter du caractère approprié du voyage. } \\
\text { - Le test cutané de dépistage de la } \\
\text { tuberculose après le voyage peut être } \\
\text { indiqué. }\end{array}$ & $\begin{array}{l}\text { Déclaration sur le voyageur } \\
\text { immunodéprimé du } \\
\text { CCMTMV (62) } \\
\text { - Évaluation du risque de } \\
\text { tuberculose et prévention } \\
\text { de cette maladie chez les } \\
\text { voyageurs du CCMTMV } \\
\text { (44) } \\
\text { Normes canadiennes pour } \\
\text { la lutte antituberculeuse } \\
\text { (chapitres } 6 \text { et } 13)(45)\end{array}$ \\
\hline
\end{tabular}

'L'Asie du Sud est définie conformément à la Classification de la Banque mondiale (67) et elle comprend l'Afghanistan, le Pakistan, l'Inde, le Népal, le Bangladesh, les Maldives, le Sri Lanka et le Bhoutan.

\section{Remerciements}

Ce résumé a été mis au point par le groupe de travail sur les voyageurs rendant visite à des amis et à des parents : Brophy J. (président), Bui Y., Crockett M., Greenaway C., McCarthy A., Jagt K., Geduld J., et Bryson M.

Membres du CCMTMV : McCarthy A. (présidente), Boggild A., Brophy J., Bui Y., Crockett M., Ghesquiere W., Greenaway C., Henteleff A., Libman M., Teitelbaum P. et Vaughan S.

Membres de liaison : Hui C. (Société canadienne de pédiatrie), Gershman M. (Centers for Disease Control and Prevention des États-Unis) et Pernica J. (Association pour la microbiologie médicale et l'infectiologie Canada).

Membres d'office : McDonald P. (Division des médicaments anti-infectieux, Santé Canada), Tepper M. (Direction de la protection de la santé de la Force, ministère de la Défense nationale), Schofield $\mathrm{S}$. (Direction de la protection de la santé de la Force, ministère de la Défense nationale), Marion D. (Centre des services de santé des Forces canadiennes, ministère de la Défense nationale).

Membre émérite : Jeanes, C. W. L. (Jusqu'en juin 2014)

\section{Conflit d'intérêts}

Aucun.

\section{Financement}

Ce travail a été appuyé par l'Agence de la santé publique du Canada.

\section{Références}

(1) Statistics Canada. International Travel Survey, Canadian Residents 2012. Custom extract for the Public Health Agency of Canada.

(2) Centers for Disease Control and Prevention. CDC Health Information for International Travel 2014. New York: Oxford University Press; 2014.

(3) Committee to Advise on Tropical Medicine and Travel. Statement on International Travellers Who Intend to Visit Friends and Relatives. 2015. (disponible en français : http://publications.gc.ca http://www.phac-aspc.gc.ca/tmp-pmv/catmat-ccmtmv/friends-amisfra.php.

(4) Barnett ED, MacPherson DW, Stauffer WM, Loutan L, Hatz CF, Matteelli A, et al. The visiting friends or relatives traveler in the 21st century: Time for a new definition. J Travel Med. 2010 May-Jun;17(3):163-170. 
(5) Behrens RH, Stauffer WM, Barnett ED, Loutan L, Hatz CF, Matteelli A, et al. Travel case scenarios as a demonstration of risk assessment of VFR travelers: Introduction to criteria and evidence-based definition and framework. J Travel Med. 2010 May-Jun;17(3):153-162.

(6) Public Health Agency of Canada. About CATMAT. 2014. http://www.phac-aspc.gc.ca/tmp-pmv/catmat-ccmtmv/index-eng.php. (disponible en français: http://www.phac-aspc.gc.ca/tmp-pmv/catmat-ccmtmv/index-fra.php).

(7) Bacaner N, Stauffer B, Boulware DR, Walker PF, Keystone JS. Travel medicine considerations for North American immigrants visiting friends and relatives. JAMA. 2004 Jun 16;291(23):2856-2864.

(8) Angell SY, Cetron MS. Health disparities among travelers visiting friends and relatives abroad. Ann Intern Med 2005 Jan 4;142(1):67-72.

(9) Baggett HC, Graham S, Kozarsky PE, Gallagher N, Blumensaadt S, Bateman J, et al. Pretravel health preparation among US residents traveling to India to VFRs: Importance of ethnicity in defining VFRs. J Travel Med. 2009 MarApr;16(2):112-118.

(10) LaRocque R, Rao S, Lawton T, Tsibris A, Schoenfeld D, Barry A, et al. Use and sources of medical information among departing international travelers to low and middle income countries at Logan International Airport-Boston, MA, 2009. Int J Inf Dis. Conference: 14th International Congress on Infectious Diseases (ICID) Miami, FL United States.Conference 2010 March 2010;14:e132.

(11) Van Herck K, Van Damme P, Castelli F, Zuckerman J, Nothdurft H, Dahlgren AL, et al. Knowledge, attitudes and practices in travel-related infectious diseases: The European Airport Survey. J Travel Med. 2004 Jan-Feb;11(1):3-8.

(12) Van Genderen PJ, Van Thiel PP, Mulder PG, Overbosch D. Trends in the knowledge, attitudes and practices of travel risk groups towards prevention of malaria: Results from the Dutch Schiphol Airport Survey 2002 to 2009. Malaria Journal. 2012;11.

(13) LaRocque RC, Deshpande BR, Rao SR, Brunette GW, Sotir MJ, Jentes ES, et al. Pre-travel health care of immigrants returning home to visit friends and relatives. Am J Trop Med Hyg. 2013;88(2):376-380.

(14) Centers for Disease Control and Prevention (CDC). CDC Health Information for International Travel 2012. New York: Oxford University Press; 2012.

(15) Behrens RH, Barnett ED. Chapter 29: Visiting Friends and Relatives. In: Keystone JS, Kozarsky PE, Freedman DO, Nothdurft H, Connor BA, editors. Travel Medicine. Second ed. USA: Mosby Elsevier; 2008. p. 291-298.

(16) Angell SY, Behrens RH. Risk assessment and disease prevention in travelers visiting friends and relatives. Infect Dis Clin North Am. 2005 Mar;19(1):49-65.

(17) McCarthy M. Should visits to relatives carry a health warning? Lancet. 2001 Mar 17;357(9259):862.

(18) Campbell H. Imported malaria in the UK: Advice given by general practitioners to British residents travelling to malaria-endemic areas. J R Coll Gen Pract. 1987 Feb;37(295):70-72.

(19) Pavli A, Maltezou HC. Malaria and travellers visiting friends and relatives. Travel Med Infect Dis. 2010 May;8(3):161168.

(20) Fanella ST, Lipkin H, Crockett ME. Presentation of pediatric malaria to a Canadian Children's Hospital. J Travel Med. 2012;19(6):391-394.

(21) Lee CS, Gregson DB, Church D, Laupland KB, Eckhardt R, Ross T, et al. Population-based laboratory surveillance of imported malaria in Metropolitan Calgary, 2000-2011. PLoS One. 2013;8(4):e60751. http://www.ncbi.nlm.nih.gov/pubmed/?term=Populationbased+laboratory+surveillance+of+imported+malaria+in+Metropolitan+Calgary

(22) Leder K, Tong S, Weld L, Kain KC, Wildersmith A, von Sonnenburg F, et al. Illness in travelers visiting friends and relatives: A review of the GeoSentinel Surveillance Network. Clin Infect Dis. 2006 Nov 1;43(9):1185-1193.

(23) McCarthy AE, Morgan CA, Prematunge C, Geduld J. Severe malaria in Canada, 2001-2013. (In press).

(24) Committee to Advise on Tropical Medicine and Travel. Canadian Recommendations for the Prevention and Treatment of Malaria. 2014. http://publications.gc.ca/collections/collection_2014/aspc-phac/HP40-102-2014-eng.pdf. (disponible en français: http://publications.gc.ca/collections/collection_2014/aspc-phac/HP40-102-2014-fra.pdf).

(25) Greenaway C, Dongier P, Boivin JF, Tapiero B, Miller M, Schwartzman K. Susceptibility to measles, mumps and rubella in newly arrived adult immigrants and refugees. Ann Intern Med. 2007 Jan 2;146(1):20-24.

(26) Greenaway C, Boivin JF, Cnossen S, Rossi C, Tapiero B, Schwartzman K, et al. Risk factors for susceptibility to varicella in newly arrived adult migrants in Canada. Epidemiol Infect. 2013 Nov 1:1-13.

(27) Parkins MD, McNeil SA, Laupland KB. Routine immunization of adults in Canada: Review of the epidemiology of vaccine-preventable diseases and current recommendations for primary prevention. Can J Infect Dis Med Microbiol. 2009;20(3):e81-90.

(28) Committee to Advise on Tropical Medicine and Travel. Statement on Pediatric Travellers. Can Commun Dis Rep. 2010;ACS-3(36):1-31. 
(disponible en français: http://www.phac-aspc.gc.ca/publicat/ccdr-rmtc/10vol36/acs-3/june-juin-2010-fra.php).

(29) Lynch MF, Blanton EM, Bulens S, Polyak C, Vojdani J, Stevenson J, et al. Typhoid fever in the United States, 19992006. JAMA. 2009;302(8):859-865.

(30) Bui Y, Trepanier S, Milord F, Blackburn M, Provost S, Gagnon S. Cases of malaria, hepatitis A and typhoid fever among VFRs, Quebec (Canada). J Travel Med. 2011 November-December 2011;18(6):373-378.

(31) Committee to Advise on Tropical Medicine and Travel. Statement on International Travellers and Typhoid. 2014. http://publications.gc.ca/collections/collection_2014/aspc-phac/HP40-98-2014-eng.pdf. (disponible en français: http://publications.gc.ca/collections/collection_2014/aspc-phac/HP40-98-2014-fra.pdf).

(32) Askling HH, Rombo L, Andersson Y, Martin S, Ekdahl K. Hepatitis A risk in travelers. J Travel Med. 2009 JulAug;16(4):233-238.

(33) Faber MS, Stark K, Behnke SC, Schreier E, Frank C. Epidemiology of hepatitis A virus infections, Germany, 20072008. Emerg Infect Dis. 2009 Nov;15(11):1760-1768.

(34) Mutsch M, Spicher VM, Gut C, Steffen R. Hepatitis A virus infections in travelers, 1988-2004. Clin Infect Dis. 2006 Feb 15;42(4):490-497.

(35) Nielsen US, Larsen CS, Howitz M, Petersen E. Hepatitis A among Danish travellers 1980-2007. J Infect. 2009 Jan;58(1):47-52.

(36) Rossi C, Shrier I, Marshall L, Cnossen S, Schwartzman K, Klein MB, et al. Seroprevalence of chronic hepatitis B virus infection and prior immunity in immigrants and refugees: A systematic review and meta-analysis. PLOS ONE. 2012;7(9).

(37) Committee to Advise on Tropical Medicine and Travel. Summary of recommendations for the prevention of viral hepatitis during travel. Can Commun Dis Rep. 2014;40(13):278-281.

(disponible en français: http://www.phac-aspc.gc.ca/publicat/ccdr-rmtc/14vol40/dr-rm40-13/dr-rm40-13-clin-2-fra.php).

(38) Committee to Advise on Tropical Medicine and Travel. Statement on Hepatitis Vaccines for Travellers. Can Commun Dis Rep. 2008;34(ACS-2):1-24.

(disponible en français: http://www.phac-aspc.gc.ca/publicat/ccdr-rmtc/08vol34/acs-2/index-fra.php).

(39) Public Health Agency of Canada. Tuberculosis in Canada 2012 - Pre-Release. 2012. http://www.phac-aspc.gc.ca/tbpc-latb/pubs/tbcan12pre/index-eng.php. (disponible en français: http://www.phac-aspc.gc.ca/tbpc-latb/pubs/tbcan12pre/index-fra.php).

(40) Ormerod LP, Green RM, Gray S. Are there still effects on Indian subcontinent ethnic tuberculosis of return visits?: A longitudinal study 1978-97. J Infect. 2001;43(2):132-134.

(41) Kik SV, Mensen M, Beltman M, Gijsberts M, Van Ameijden EJC, Cobelens FGJ, et al. Risk of travelling to the country of origin for tuberculosis among immigrants living in a low-incidence country. Int J Tuberc Lung D. 2011;15(1):38-43.

(42) Saiman L, San Gabriel P, Schulte J, Vargas MP, Kenyon T, Onorato I. Risk factors for latent tuberculosis infection among children in New York City. Pediatrics. 2001;107(5):999-1003.

(43) Lobato MN, Hopewell PC. Mycobacterium tuberculosis infection after travel to or contact with visitors from countries with a high prevalence of tuberculosis. Am J Respir Crit Care Med. 1998 Dec;158(6):1871-1875.

(44) Committee to Advise on Tropical Medicine and Travel. Risk Assessment and Prevention of Tuberculosis Among Travellers. Can Commun Dis Rep. 2009;35(ACS-5):1-20.

(disponible en français: http://www.phac-aspc.gc.ca/publicat/ccdr-rmtc/09vol35/acs-dcc-5/index-fra.php).

(45) Public Health Agency of Canada. Canadian Tuberculosis Standards. 7th ed. Ottawa (ON): Public Health Agency of Canada, Canadian Lung Association/Canadian Thoracic Society; 2014.

(disponible en français: http://www.phac-aspc.gc.ca/tbpc-latb/pubs/tb-canada-7/assets/pdf/tb-standards-tb-normesch6-fra.pdf).

(46) Boggild AK, Yohanna S, Keystone JS, Kain KC. Prospective analysis of parasitic infections in Canadian travelers and immigrants. J Travel Med. 2006 May-Jun;13(3):138-144.

(47) Vivancos R, Abubakar I, Hunter PR. Foreign travel, casual sex and sexually transmitted infections: systematic review and meta-analysis. Int J Infect Dis 2010 Oct;14(10):e842-51.

(48) Kramer MA, van den Hoek A, Coutinho RA, Prins M. Sexual risk behaviour among Surinamese and Antillean migrants travelling to their countries of origin. Sex Transm Infect. 2005 Dec;81(6):508-510.

(49) Fenton KA, Chinouya M, Davidson O, Copas A, MAYISHA research team. HIV transmission risk among sub-Saharan Africans in London travelling to their countries of origin. AIDS. 2001 Jul 27;15(11):1442-1445.

(50) Matteelli A, Schlagenhauf P, Carvalho ACC, Weld L, Davis XM, Wilder-Smith A, et al. Travel-associated sexually transmitted infections: An observational cross-sectional study of the GeoSentinel surveillance database. Lancet Infect Dis. 2013;13(3):205-213 2013.

(51) Fenner L, Weber R, Steffen R, Schlagenhauf P. Imported infectious disease and purpose of travel, Switzerland. Emerg Infect Dis. 2007 Feb;13(2):217-222. 
(52) Committee to Advise on Tropical Medicine and Travel. Statement on Travellers and Sexually Transmitted Infections. Can Commun Dis Rep. 2006;32(ACS-5):1-24.

(disponible en français: http://www.phac-aspc.gc.ca/publicat/ccdr-rmtc/06vol32/acs-05/index-fra.php).

(53) Mclnnes RJ, Williamson LM, Morrison A. Unintentional injury during foreign travel: A review. J Travel Med. 2002 NovDec;9(6):297-307.

(54) Lunetta P. Injury deaths among Finnish residents travelling abroad. Int J Inj Contr Saf Promot. 2010 Sep;17(3):161168.

(55) MacPherson DW, Gushulak BD, Sandhu J. Death and international travel--the Canadian experience: 1996 to 2004 . J Travel Med. 2007 Mar-Apr;14(2):77-84.

(56) Committee to Advise on Tropical Medicine and Travel. Statement on Risk of Injury and Travel. Can Commun Dis Rep. 2010;36(ACS-13):1-14.

(disponible en français: http://www.phac-aspc.gc.ca/publicat/ccdr-rmtc/10vol36/acs-13/index-fra.php).

(57) Valerio L, Roure S, Sabria M, Balanzo Xd, Moreno N, MartinezCuevas O, et al. Epidemiologic and biogeographic analysis of 542 VFR traveling children in Catalonia (Spain). A rising new population with specific needs. J Travel Med. 2011;18(5): 304-309.

(58) Han P, Yanni E, Jentes ES, Hamer DH, Chen LH, Wilson ME, et al. Health challenges of young travelers visiting friends and relatives compared with those traveling for other purposes. Pediatr Infect Dis J. 2012;31(9):915-919.

(59) Hagmann S, Neugebauer R, Schwartz E, Perret C, Castelli F, Barnett ED, et al. Illness in children after international travel: Analysis from the GeoSentinel Surveillance Network. Pediatrics. 2010 May;125(5):e1072-80.

(60) Hunziker T, Berger C, Staubli G, Tschopp A, Weber R, Nadal D, et al. Profile of travel-associated illness in children, Zurich, Switzerland. J Travel Med. 2012;19(3):158-162.

(61) Crockett M, Hui C, Kuhn S, Ford-Jones L, Grondin D, Keystone J. Travel-related illnesses among pediatric VFRs in Canada. American Society of Tropical Medicine and Hygiene 60th Annual Meeting Dec. 4 - 8, 2011;Philadelphia, PA, USA(No. 968).

(62) Committee to Advise on Tropical Medicine and Travel. The Immunocompromised Traveller. Can Commun Dis Rep 2007;33(ACS-4):1-24.

(disponible en français: http://www.phac-aspc.gc.ca/publicat/ccdr-rmtc/07vol33/acs-04/index-fra.php).

(63) Committee to Advise on Tropical Medicine and Travel. Statement on Older Travellers. Can Commun Dis Rep. 2011;37(ACS-2):1-24.

(disponible en français: http://www.phac-aspc.gc.ca/publicat/ccdr-rmtc/11vol37/acs-2/index-fra.php).

(64) Committee to Advise on Tropical Medicine and Travel. Statement on Personal Protective Measures to Prevent Arthropod Bites. Can Commun Dis Rep. 2012;38(ASC-3):1-18.

(disponible en français: http://www.phac-aspc.gc.ca/publicat/ccdr-rmtc/12vol38/acs-dcc-3/index-fra.php).

(65) Public Health Agency of Canada. Canadian immunization guide. Cat.: HP40-3/2014E ed. Ottawa (ON): Public Health Agency of Canada; 2014.

(disponible en français: http://www.phac-aspc.gc.ca/publicat/cig-gci/assets/pdf/p01-fra.pdf).

(66) World Health Organization [Internet]. Hepatitis B, countries or areas at risk. 2012. http://gamapserver.who.int/mapLibrary/Files/Maps/Global_HepB_ITHRiskMap.png.

(67) World Bank [Internet]. South Asia. 2014. http://www.worldbank.org/en/region/sar. 